Supplemental information

\title{
A method for detecting circulating tumor cells based on measurement of single cell metabolism in droplet-based microfluidics
}

Fabio Del Ben ${ }^{\dagger} *$, Matteo Turetta ${ }^{\dagger}$, Giorgia Celetti, Aigars Piruska, Michela Bulfoni, Daniela Cesselli, Wilhelm T.S. Huck*, Giacinto Scoles*

\section{Experimental Section}

Microfluidic circuit fabrication: $25 \mu \mathrm{m}$ thick layer of SU8-2025 was spun on silicon wafer, baked, exposed through transparency mask, baked again and developed according to manufacturer instructions (MicroChem corp.). Sylgard 184 (PDMS) prepolymer and crosslinking agent (Dow Corning) were mixed at a mass ratio of $10: 1(\mathrm{w} / \mathrm{w})$; a mixture was poured onto a master, degassed and cured at $65^{\circ} \mathrm{C}$ for at least $2 \mathrm{~h}$. The replica was detached from master and reservoirs were bored using a blunt hypodermic needle. A PDMS replica was washed in soapy water and ethanol, and blow dried with nitrogen. A clean glass slide and a clean PDMS replica were treated with oxygen plasma and bonded. The device was silanized with 1\% (Tridecafluoro-1,1,2,2-Tetrahydrooctyl)-1-Trichlorosilane (Sigma-Aldrich) in FC-40 (3M), fluorinated oil, which was introduced into microfluidic channels (enough to completely wet whole microfluidic network) and then the device was kept at $95^{\circ} \mathrm{C}$ for at least $30 \mathrm{~min}$. To fabricate a reservoir for an emulsified sample a brass cone (10 mm in dia. and $5 \mathrm{~mm}$ tall; 130 $\mu \mathrm{L}$ volume) was placed directly on silicon wafer and replicated together with photolithographically defined features.

Cells: Cell lines were a courtesy of Di Loreto lab, Hubrecht lab, and Colombatti lab. White blood cells (WBCs) were obtained by lysing whole blood with lysis solution (BD) according to manufacturer's protocol.

Lactate enzymatic assay: A cell suspension and a lactate assay mixture (Cell Technology, Inc.) were mixed and immediately emulsified using a chip with 3 inlets. Thus cells were exposed to lactate assay mixture only during emulsification. In addition the emulsification step was performed at $4^{\circ} \mathrm{C}$ to slow down cancer cell metabolism and to prevent them from lactate secretion in the bulk. Flow rates of cell suspension and lactate assay mixture was kept at ratio $1: 1$. Droplets were incubated for $15 \mathrm{~min}$ and imaged on an epifluorescence microscope.

Widefield fluorescence imaging (lactate assay, pHRodo green experiments): An inverted epifluorescence microscope (Olympus IX81) was equipped with xCite 120Q lamp (Lumen Dynamics Group Inc.), resorufin and FITC filter sets (Semrock) and iXon 897 camera (Andor). An aliquot of processed sample was pipetted on a microscope glass slide and covered with a cover slip to prevent evaporation during imaging.

High throughput detection with SNARF-5F. Setup: An inverted microscope (Olympus IX70) was used to analyze flowing droplets one by one. A laser (488 Argon-ion Cyonics) beam was expanded ( $2 \mathrm{x}$ ) and focused down with a cylindrical lens crossing orthogonally the microfluidic channel. The fluorescence signal of excited droplets was collected with a 40x objective (Olympus LUCPlanFLN, 40x/0.60), split with 
dichroic filter (DLP555, Semrock) and detected through bandpass filters (579/34 630/38) by Photo Multiplier Tubes (PMTs) (H957-15, Hamamatsu). Signal went through a transimpedance amplifier with $1 \mathrm{~V} / \mathrm{uA}$ gain and was detected by the acquisition system (National Instruments CRIO-9024, analog input module NI9223) with a $10^{5} \mathrm{~Hz}$ scan rate. The acquisition system was driven by LABView in house written software. The software detects all data-points of a droplet over a set threshold and computes in real time averaged values; it also provides trigger pulse for image capture on a camera. Liquids were pumped using neMESYS (Cetoni) syringe pumps.

Droplet production: Monodisperse droplets were generated in chips with $20 \mu \mathrm{m}$ wide T-junction. Continuous phase: $2 \%\left(\mathrm{w} / \mathrm{w}\right.$ ) surfactant (Krytox-Jeffamine-Krytox A-B-A triblock copolymer) ${ }^{1}$ in HFE7500 (3M) Dispersed phase: cell suspension in HBSS or Joklik's modified EMEM containing 15\% Optiprep and $4 \mu \mathrm{M}$ to $10 \mu \mathrm{M}$ SNARF 5F. Flow rates were set such as continuous phase flow was at least 2 times higher than a flow rate of a dispersed phase; a typical flow rate for a disperse phase was $300 \mu \mathrm{L} / \mathrm{h}$ and $600 \mu \mathrm{L} / \mathrm{h}$ for a continuous phase.

A549 quantitation. Cultured cells were washed with PBS, trypsinized and transferred into medium (typical concentration 500,000 to 1000,000 cell/mL). Cells were spun down and resuspended in the running buffer - 2\% BSA in Joklik's modified EMEM ( $\mathrm{pH}$ 7.4). If lower concentration of A549s was required, cell suspension was diluted to 1000 cell/mL in Joklik's modified EMEM. $100 \mu \mathrm{L}$ of sample solution was obtained by mixing cell suspension, SNARF-5F stock (conc. $1 \mathrm{mM}$ in DI water and ethanol $(1: 1 \mathrm{v} / \mathrm{v}))$ and running buffer. A fraction of obtained solution was used to verify A549 concentration in a counting chamber. To obtain A549 samples with WBCs a 1 to $2 \mathrm{M}$ cell/mL WBC suspension in the running buffer was prepared. The obtained WBC suspension was used together with the previously prepared A549 suspension and the SNARF-5f stock to obtain samples. A549 samples (with or without WBC) were emulsified at the flow rate of $300 \mu \mathrm{L} / \mathrm{h}(600 \mu \mathrm{L} / \mathrm{h}$ for oil), collected in cone reservoir and incubated for 10 to 20 minutes at $37^{\circ} \mathrm{C}$. Tubing with an internal diameter $100 \mu \mathrm{m}$ was used to connect a cone with an inlet of readout device. Droplets were reinjected from cone device directly into a readout device (50 to $100 \mu \mathrm{L} / \mathrm{h}$ for droplets; 300 to $500 \mu \mathrm{L} / \mathrm{h}$ for spacer oil). Detected cancerous cells were verified with images acquired for droplets with reduced $\mathrm{pH}$. The visual inspection was critical to properly quantify spiked tumor cells. Debris, particulates and large cell clusters were typical false positives that could be easily discriminated from cancer cells based on their transmission image.

CD45 immunomagnetic depletion: We followed manufacturer's protocol for CD45+ depletion using Miltenyi human CD45 Microbeads, MidiMACS ${ }^{\text {TM }}$ Separator, MultiStand and LD Column.

Patient protocol: $2 \mathrm{~mL}$ of whole blood from metastatic cancer patients, taken with a venous puncture and collected in EDTA tubes, was lysed with BD lysis RBC lysis solution, depleted of CD45+ fraction with CD45 magnetic beads and LD columns (Miltenyi), and subsequently CD45-Alexa488 stained (BD - $4{ }^{\circ} \mathrm{C}$ incubation for 20 minutes). The prepared cell sample was resuspended in incubation medium and $15 \%$ Optiprep for a final volume of 50-70uL. Washing steps are performed with centrifugations at $300 \mathrm{~g} \times 5$ minutes at room temperature.

\footnotetext{
${ }^{1}$ V.Chokkalingam, J.Tel, F.Wimmers, X.Liu, S.Semenov, J.Thiele, C.G.Figdor, W.T.S.Huck, Lab Chip 2013,13, 47404744, doi: 10.1039/C3LC50945A
} 
Patient spike protocol: A549 cells were spiked into 1-2mL of whole blood from healthy donor and "patient protocol" was followed.

Viability assay

$1 \mathrm{mg}$ Calcein AM was dissolved in $1 \mathrm{~mL}$ DI WT to yield stock solution with $1 \mathrm{mM}$ concentration. $5 \mu \mathrm{L}$ of stock solution were diluted in $5 \mathrm{~mL}$ of PBS to yield $1 \mu \mathrm{M}$ solution.

SYTOX AADvanced (ThermoFisher Scientific, S-10349) dead cell staining solution was prepared according to instruction - dye was dissolved in $100 \mu \mathrm{L}$ of DMSO.

Similarly to sample preparation A549 cells were trypsinized, suspended in the culture medium and counted. A549s were resuspended at a concentration $1 \mathrm{e} 61 / \mathrm{mL}$ in the running buffer (2\% BSA in Joklik modified medium, $\mathrm{pH}$ 7.4) and emulsified. The obtained emulsion was collected in a vial and incubated at $37^{\circ} \mathrm{C}$. At desired time intervals a $20 \mu \mathrm{L}$ aliquot of emulsion was taken for a viability assay.

$20 \mu \mathrm{L}$ of emulsion was transferred to a vial and a) $100 \mu \mathrm{L}$ of $20 \%$ PFO in HFE 7500, and b) $100 \mu \mathrm{L}$ of running buffer were added to it. The vial was gently taped until majority of droplets coalesce into few large droplets. The vial was spun down for 30 to 60 seconds to complete the process. (2 clearly distinguished bulk phases were observed. If there were still droplets remaining, oil phase was removed, a new aliquot of PFO in HFE 7500 added and a centrifugation step repeated.) The majority of oil phase was removed and 20 to $40 \mathrm{uL}$ of water phase were collected with a pipet. A pipet tip was positioned as close as possible to the oil / water interface while ensuring that only water phase is collected.

The recovered cell suspension was placed in observation chamber and $100 \mathrm{uL}$ of Calcein AM working solution $(1 \mu \mathrm{M})$ and $1 \mathrm{uL}$ of SYTOX AADvanced solution was added to it. Suspension was incubated for 30 min at room temperature. Suspension was diluted with $~ 0.5 \mathrm{~mL}$ of PBS and imaged on inverted microscope (standard FITC cube was used for Calcein AM and cube with excitation at $561 \mathrm{~nm}$ and emission at $676 \mathrm{~nm}$ was used for SYTOX AADvanced).

\section{Supplemental results}

Cell occupancy in droplets
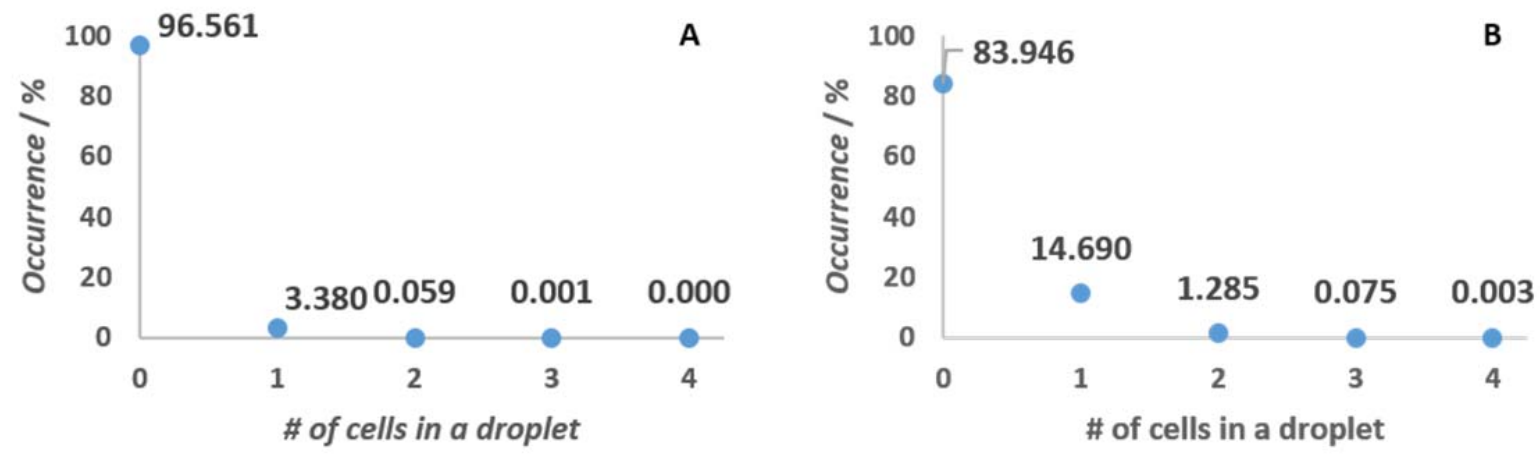

Figure S1 Distribution of cell occupancy in droplets. Distributions shown for emulsification of $1000000 \mathrm{cell} / \mathrm{mL}$ (A) and 5000000 cell/mL (B). By far the majority of droplets are empty, but more than 90\% of droplets containing cells have only 1 cell per droplet. 


\section{Detection of lactate secretion via pHRodo Green}

A sample of A549s (or WBCs) was resuspended in Joklik's modified EMEM; pHRodo Green was added to yield $5 \mu \mathrm{M}$ final concentration and obtained mixture was emulsified and incubated at $37^{\circ} \mathrm{C}$ for $30 \mathrm{~min}$. Comparison of empty and droplets containing tumor or WBCs is shown in Figure S2.
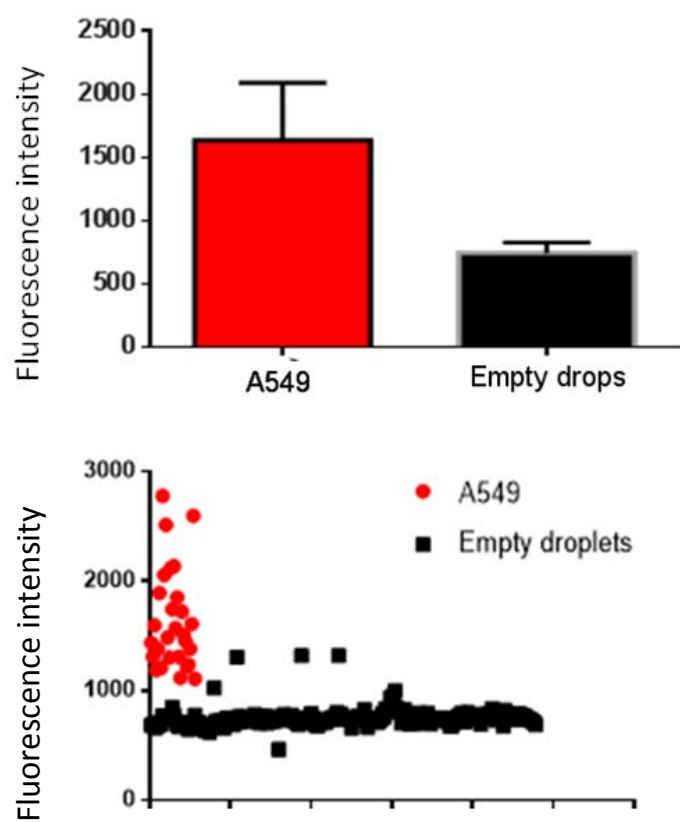
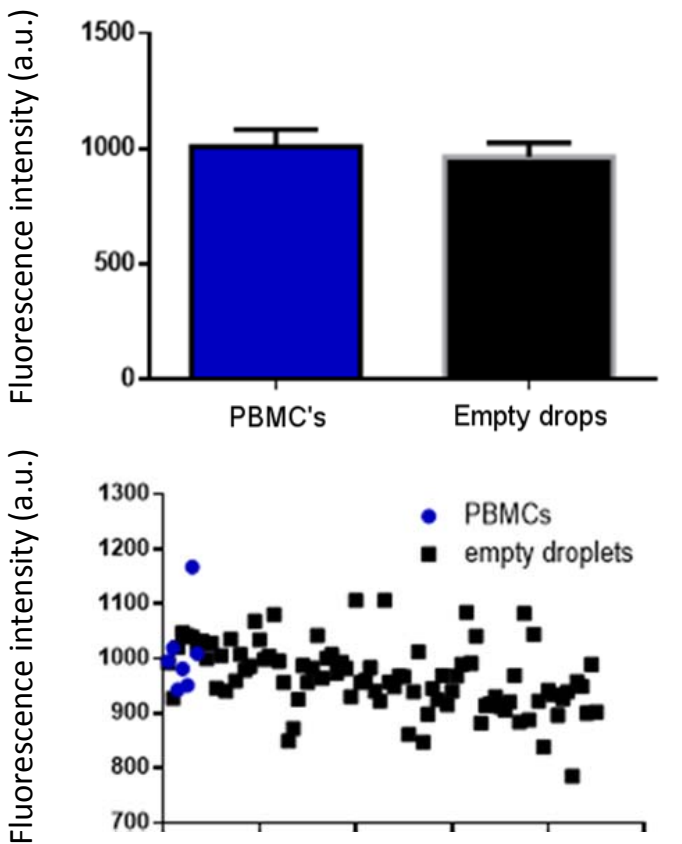

Figure S2 Detection of lactate secretion via $\mathrm{pH}$ measurement. pHrodo Green dye was used to indicate pH changes. Results are comparable to lactate assay and confirm the difference between cancer cells and white blood cells.

\section{SNARF-5F calibration}

A set of Joklik's modified EMEM solutions was prepared and titrated to various pHs between 7.4 and 5 . These solutions were emulsified and ratio of obtained droplets detected similarly to A549 samples.

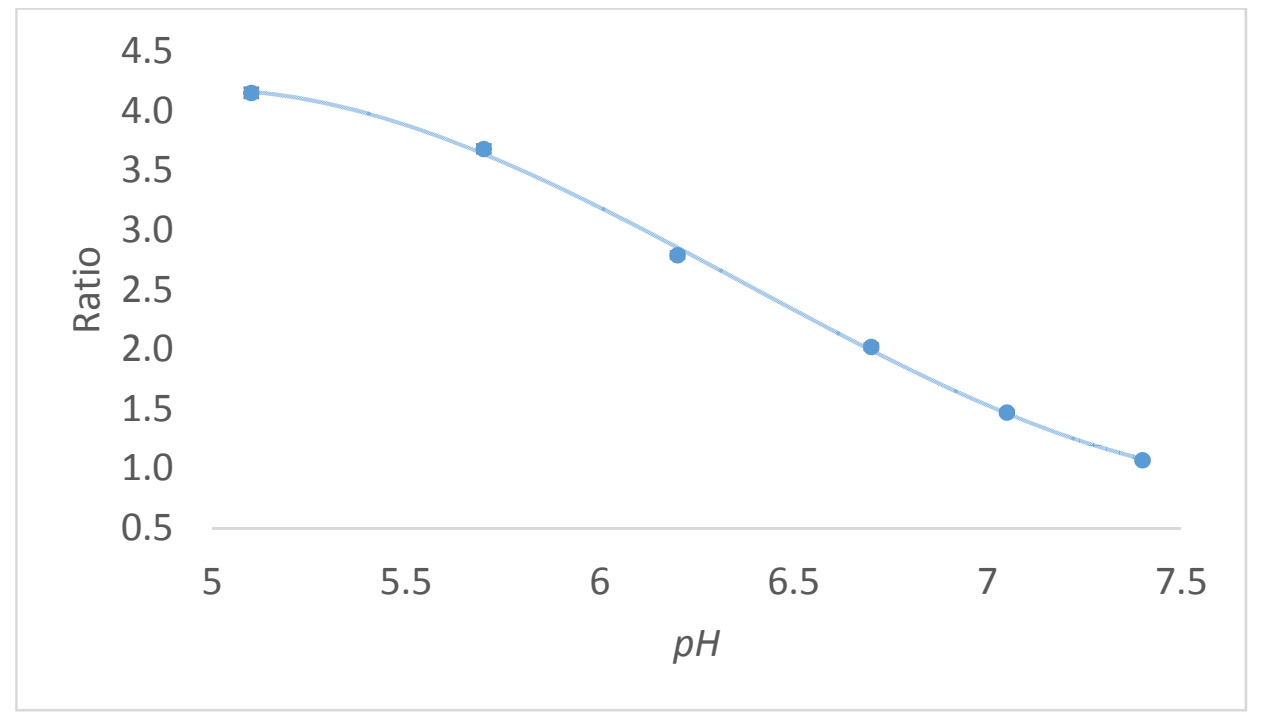


Figure S3 Calibration of SNARF-5F response. Ratio between fluorescence intensity at $580 \mathrm{~nm}$ and intensity at $630 \mathrm{~nm}$.

\section{SNARF-5F performance}

We have chosen the free carboxylic acid version of SNARF $5 \mathrm{~F}$ as an extracellular $\mathrm{pH}$ reporter. This dye is cell impermeant (see https://www.thermofisher.com/order/catalog/product/S23922). The raw fluorescent traces show that the peak shape is not significantly altered by presence of a cell (see Figure S4). Here several empty droplets and droplets containing stained WBCs are seen, but the peak shape is almost identical. This observation is consistent with the assumption that the dye is present only in an extracellular medium.

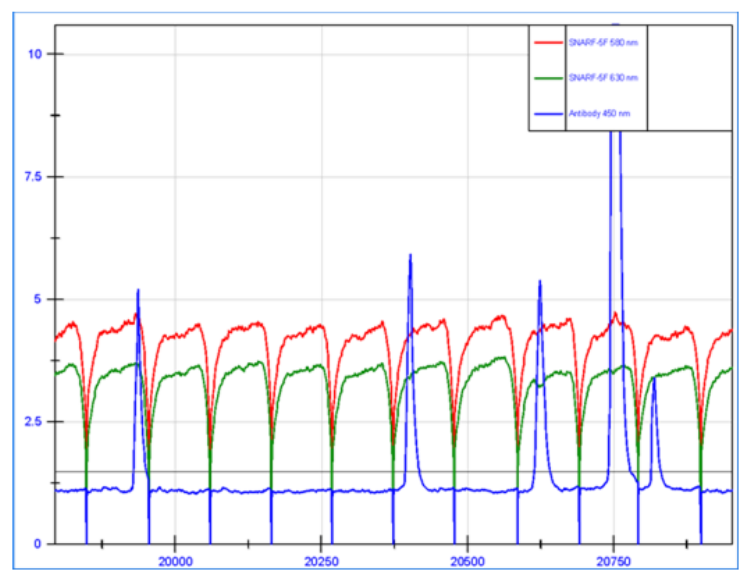

Figure S4 An example of raw data trace. Red trace - SNARF $5 F 580 \mathrm{~nm}$, green - SNARF $5 F \quad 630 \mathrm{~nm}$, blue - CD45 stain at $450 \mathrm{~nm}$. The blue peaks indicate the presence of CD45 stained WBC in droplets. 


\section{Evaluation of acidification dynamics}

TOV21G cancer cell line was evaluated. All samples were processed similarly to protocol described above. Incubation time for different samples were varied from 2 to 90 minutes.
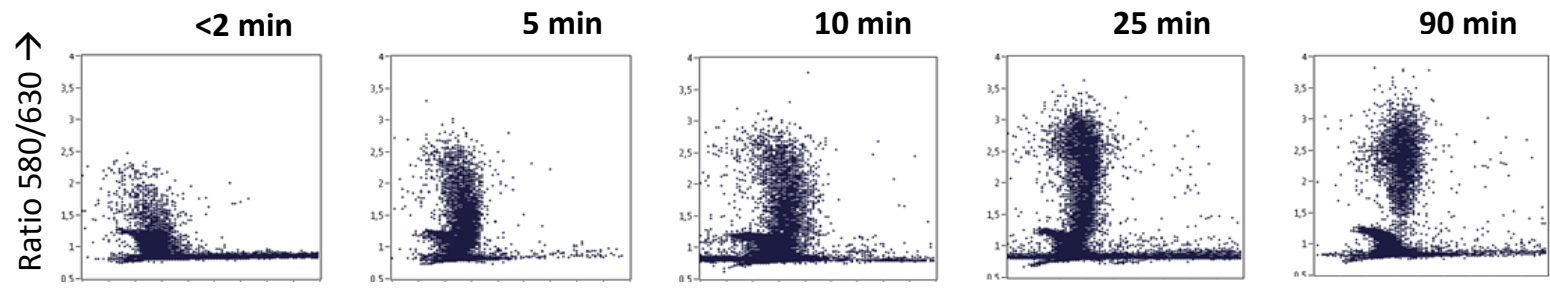

Droplet number $\rightarrow$

Kinetics of $\mathrm{pH}$ change

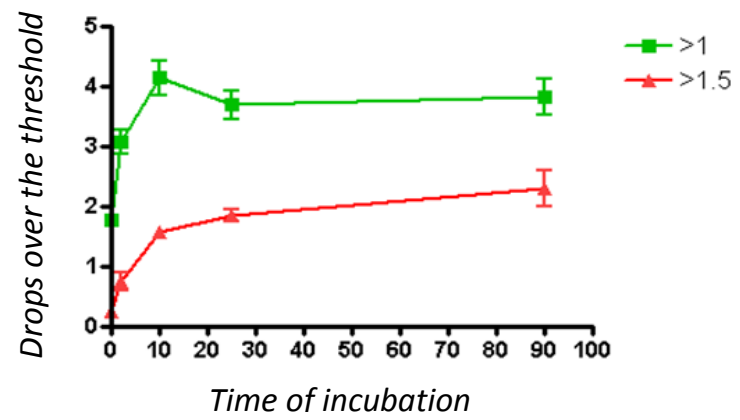

Figure S5. (Top panels) Dot plots showing a population of droplets becoming increasingly acid over time. (Bottom panel) $A$ fraction of droplets reaching certain ratio (>1 - green trace; $>1.5$ - red trace) 


\section{Extracellular acidification, evaluation of various cell lines}

To demonstrate that this is a general method for detection of cancer cells, we tested several different cancer cell lines, both $\operatorname{EpCAM(+)}$ and (-) including. These samples are processed similarly to A549 samples described in Experimental section.

MDA-MB-231

Triple negative breast

cancer

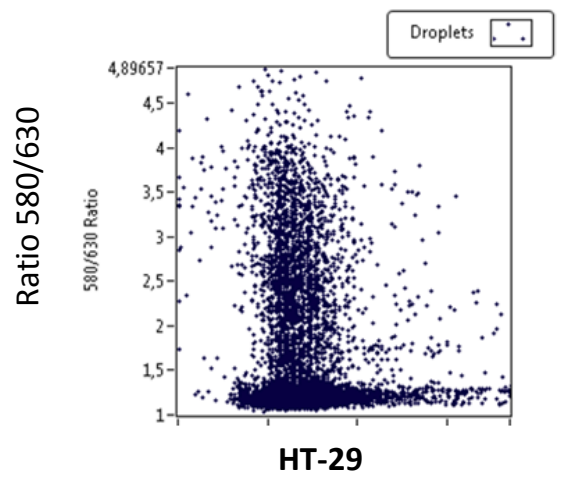

Colorectal adenocarcinoma

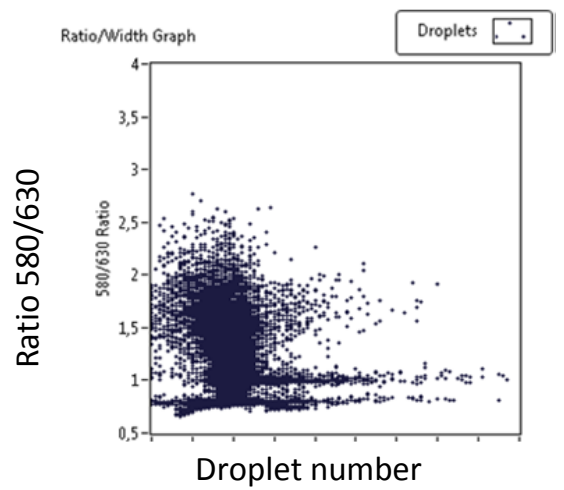

MDA-MB-453

Breast cancer

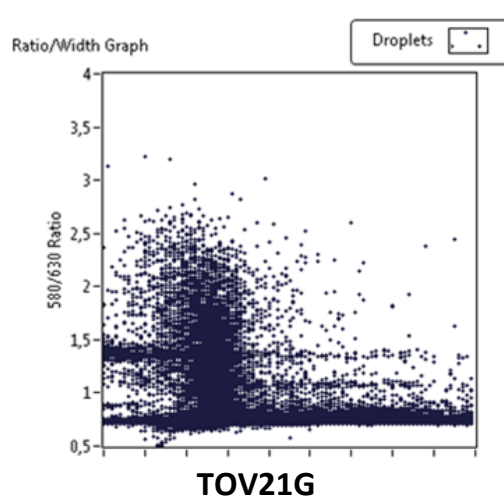

Ovarian

adenocarcinoma/clear cell

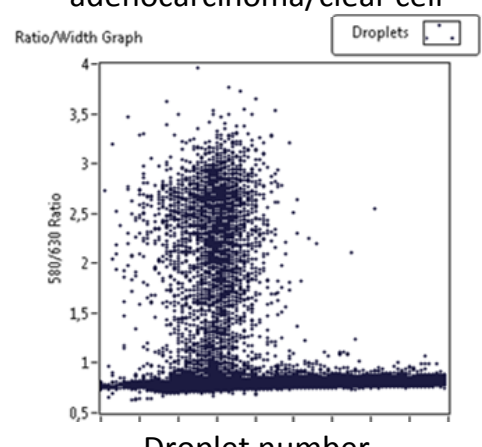

Droplet number
MCF -7

Breast cancer

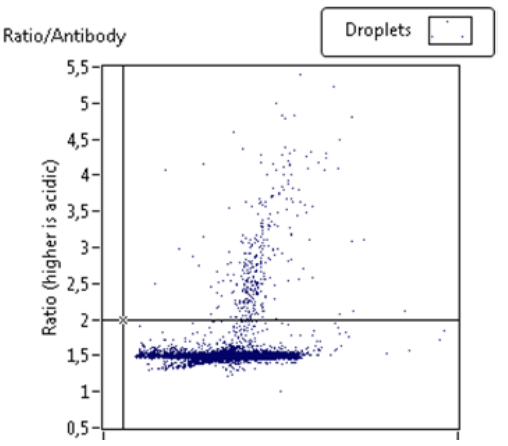

White blood cells

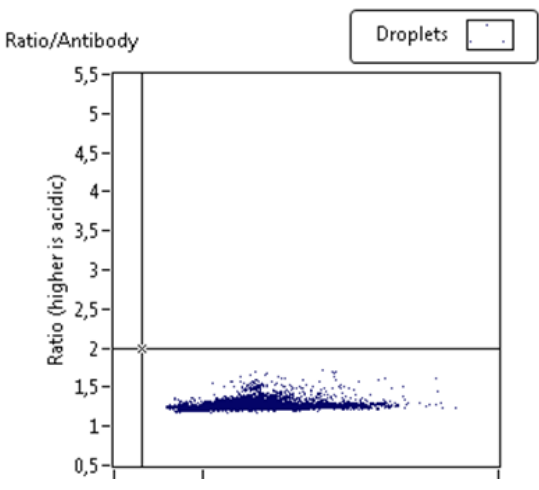

Droplet number

Figure S6 Metabolic response of selected cancer cell lines. WBC response is given for comparison. 
Cancer cells pre-stained and mixed with WBCs.

Figure S7 shows three replicate experiments where all tumour cells were stained using Calcein Violet $\mathrm{AM}-\mathrm{a}$ viability staining not affecting cell behavior - prior to mixing. Most acid-positive droplets can be found in the Calcein channel, confirming the excellent selectivity of the assay. We detected only rare acid-positive Calcein-negative drops, and by visualizing them we found out they were clusters of 10+ WBCs or junk artifacts.
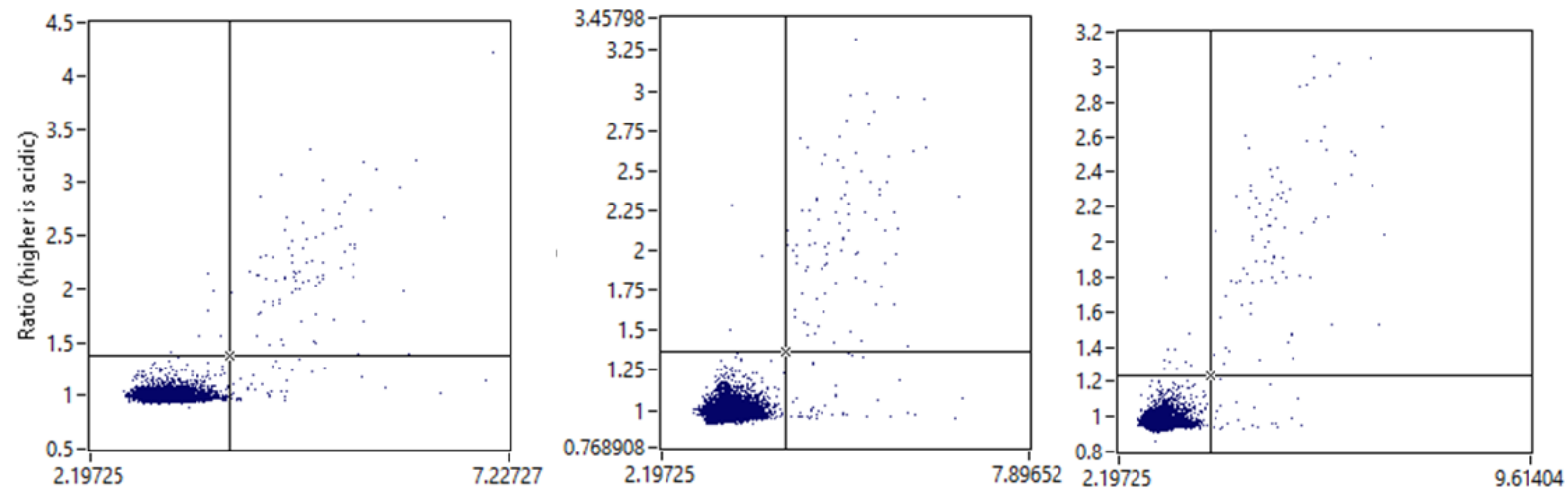

Figure S7. Dot plots of Calcein Violet pre-stained FaDu cancer cells in a mix with WBCs. FaDU 1:50 WBCs in Joklik 0,1\% BSA, Optiprep 15\%. 20.000 FaDU in 1.000.000 WBCs. 30 min incubation time. 



\section{Estimate of a secretion rate}

In order to estimate secretion rates within microdroplets we use observed $\mathrm{pH}$ change and a buffer capacity of the running buffer. The buffer capacity can be estimated in a titration experiment. We titrated $10 \mathrm{~mL}$ of running buffer (2\% BSA in Joklik medium) with $10 \mathrm{mM} \mathrm{HNO}_{3}$ solution (see Figure S8).

The obtained titration curve is expressed as a $\mathrm{pH}$ value with respect of added amount of acid per volume of the sample and is shown in Figure $\mathrm{S6}$, the buffer capacity $(\beta)$ is estimated from slope of this curve and is $-0.007 \mathrm{~mol} / \mathrm{L}$. For cancer cells we observe a ratio increase from 1 to 2 that corresponds to $\mathrm{pH}$ change $(\Delta \mathrm{pH})$-0.8. Drop with a radius of 20 microns has volume $(\mathrm{V})$ of $32 \mathrm{pL}$. Secretion rate could be calculated as:

$$
r=\frac{V \beta \Delta p H}{t N}
$$

For 20 min of incubation and single cell occupancy $(\mathrm{N}=1)$ the secretion rate is estimated $1.5 \mathrm{e}-16$ $\mathrm{mol} / \mathrm{cell} / \mathrm{s}$. This value corresponds reasonably well with previously published secretion value $1 \mathrm{e}-16$

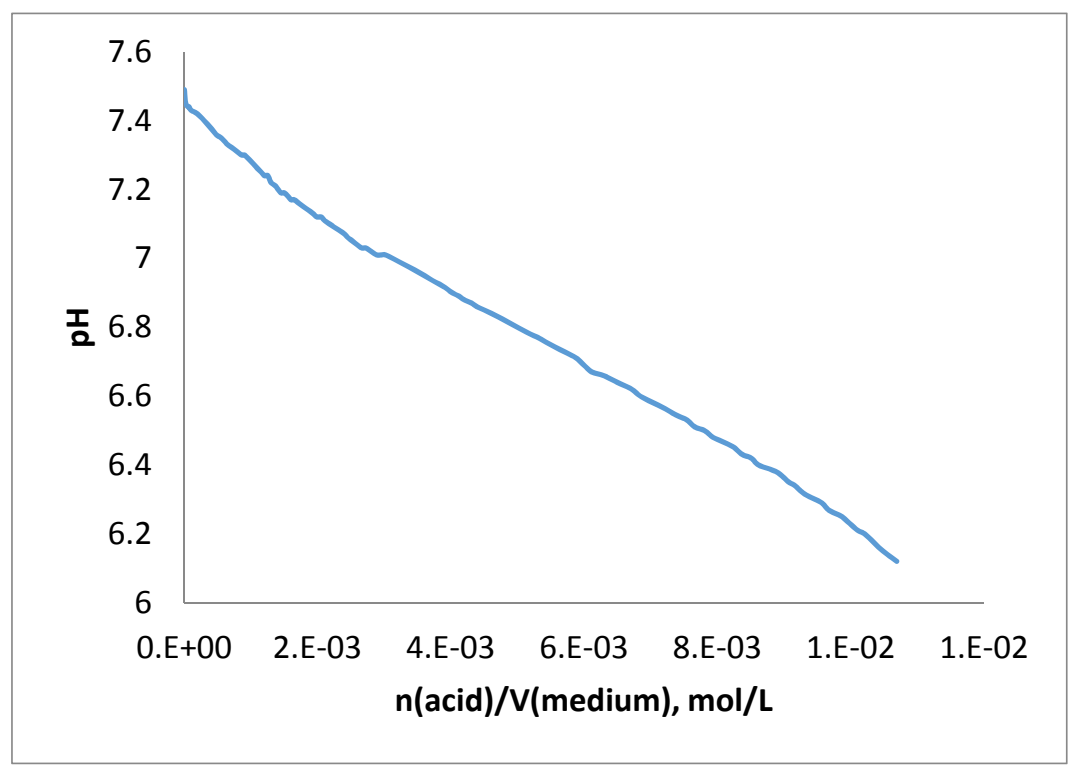

mol/s/cell \{ref: DeBerardinis, R. J., et al. PNAS 2007, 104, 19345\}.

Figure S8. A titration curve of Joklik medium with $10 \mathrm{mM} \mathrm{HNO}_{3}$. 


\section{A549 analysis}

a

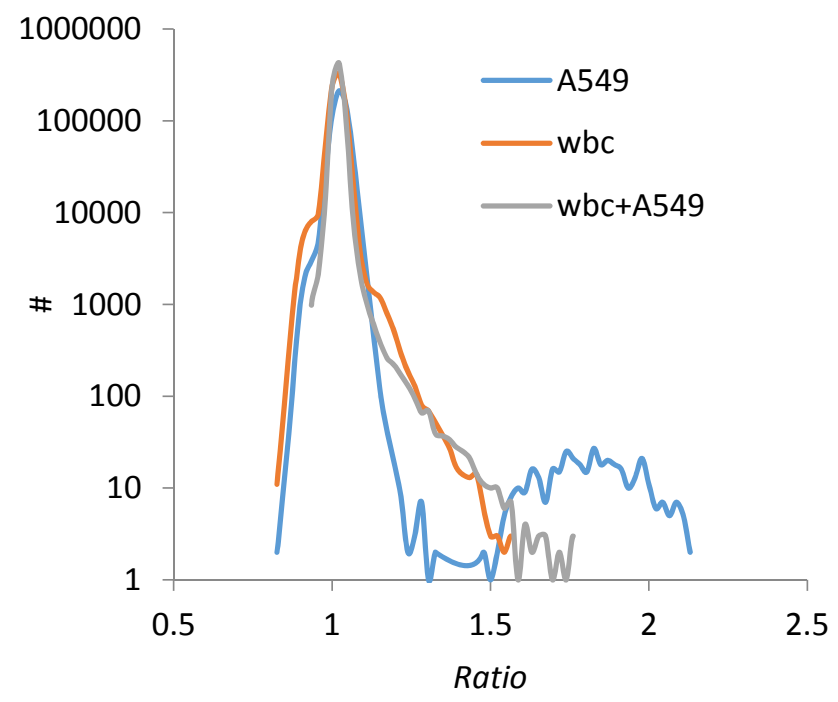

C b
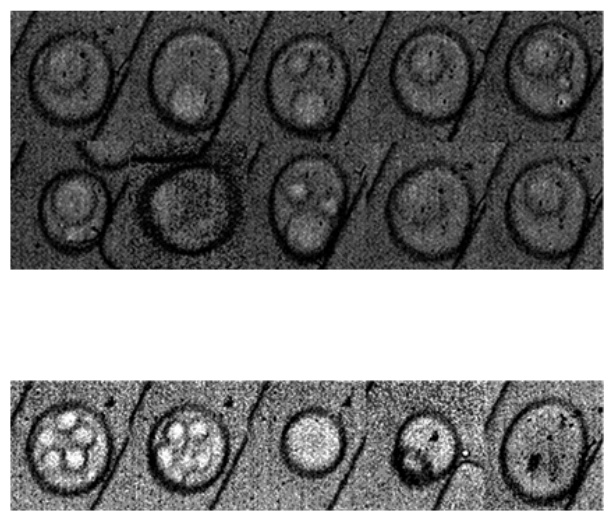
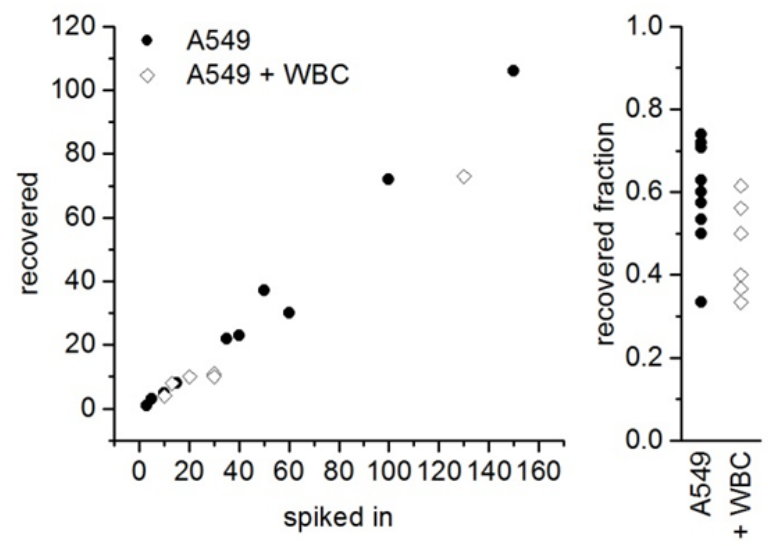

Figure S9 (a) Ratio distribution of three different samples: A549s only;) WBCs only; and A549s with WBCs. b) Micrographs used for cell identification: (top right) A549s and (bottom right) false positives - wbc clusters, small empty droplets and droplets containing debri.c) fraction of $A 549$ recovered from spike in samples.

Figure S9a shows ratio distributions for three different samples: A549s only; WBCs only and A549s with WBCs. In A549 data set empty and cancer cell containing droplets are clearly separated. Even when large number of WBCs are added for analysis majority of analyzed droplets are still empty. Cells are stochastically incorporated in droplets and in order to obtain single cell occupancy average cell concentration in bulk has to be kept below 1 cell per droplet. Droplets occupied by WBCs show somewhat increase in acidity and appear as an increased shoulder between ratios 1 and 1.5 (see WBC and $W B C+A 549$ traces). The difference between $W B C$ and $W B C+A 549$ curves is very subtle. In the case of WBC+A549 data there is elongated extension of the shoulder above ratio 1.5. This extension is clearly overlapped with droplet population containing A549s. We can visually verify presence of cancer cells in these droplets. However we found that ratio alone is not sufficient to make a conclusive determination if droplet contains cancerous cell or no. We are looking for rare events and there are others sources that 
could displaying elevated ratios, but are not cancerous cells. For example (see Figure S9b) we have observed that in some WBC samples there is more cluster formation and large clusters having 10 or more cells can produce similar ratios to cancerous cells. Also small particles, most likely originated from silanization process, can shift ratio to higher values. Thus making visual inspection critical for final classification. While the visual inspection seem like laborious process it requires only inspection on order of 10 to 100 images per $10^{6}$ droplets, a task easily accomplished in post analysis phase by an analyst and with possibility being fully automated.

Figure 9c reproduces Figure 3D from the main text, but also adds information on the fraction of A549's recovered in the various samples. 


\section{CD45 depletion and staining on patients}

We showed dot plot of lysed blood from healthy donor, whole blood from healthy donor, cancer cell line spike and colorectal cancer patient depleted blood. Please note spiked sample and patient sample display a significant population Acid(+), CD45(-).

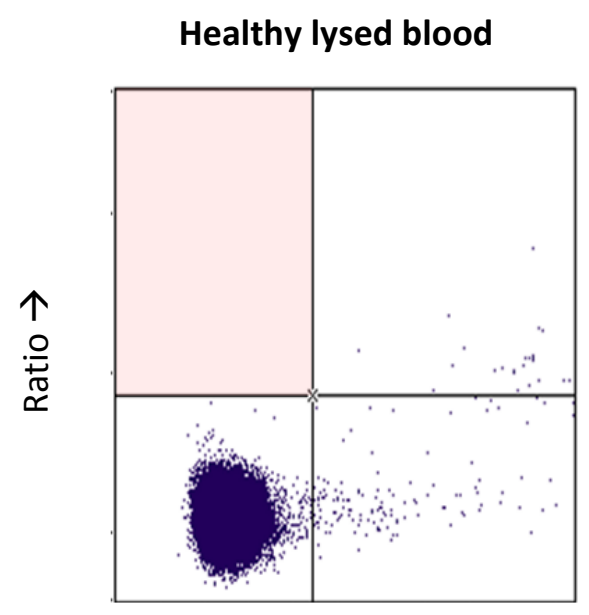

Cancer cell line

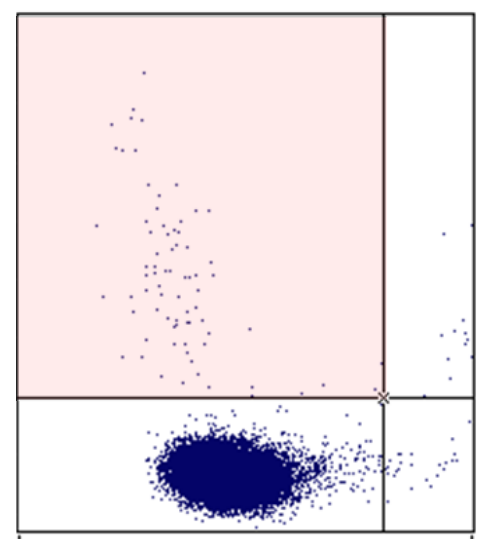

\section{Colorectal cancer patient}

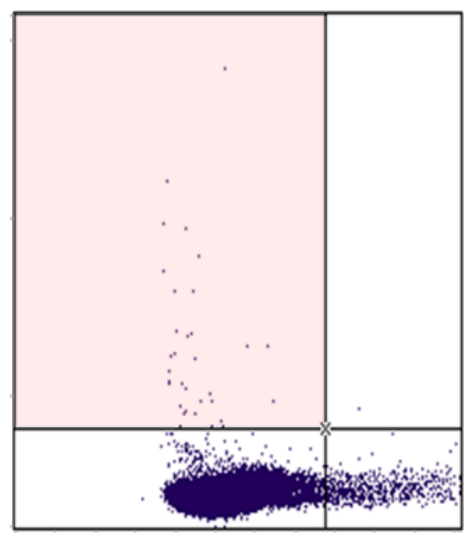

\section{$\mathrm{CD} 45 \rightarrow$}

Figure S10. Dot plot from healthy donor lysed blood, cancer cell line spike and colorectal cancer patient. CD45 on X-axis, Ratio on $Y$-Axis. Acid(+) CD45(-) population (coloured top-right quadrant) is present significantly more in spiked and patient samples than in healthy sample. Gating is qualitative and positioned to include empty droplet population in the bottom-left quadrant as internal negative control for both Ratio and CD45. 


\section{Metastatic cancer patient pictures}

In Figure S11-A, CD45(+) cells are shown for reference. Acid(+) CD45(-) cells- a small cell-cluster and a cell with size comparable to WBCs - were found in a pancreatic cancer patient (Figure S11-B). In Figure S11-C a cell larger than WBCs (20.8um) is seen together with a white blood cell. Unfortunately the cells were enclosed together, so the droplet shows CD45 positivity. Some cells in cancer patients have a morphology that is not compatible with WBCs.
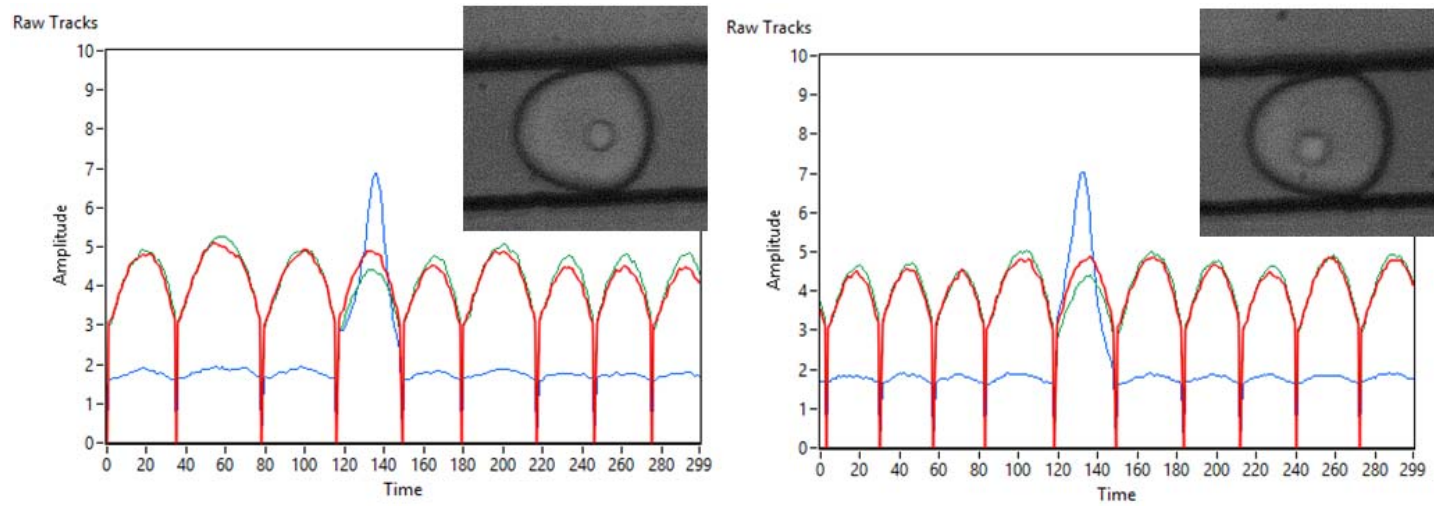

Figure S11-A. Raw tracks and picture of WBCs, showing CD45 positivity.

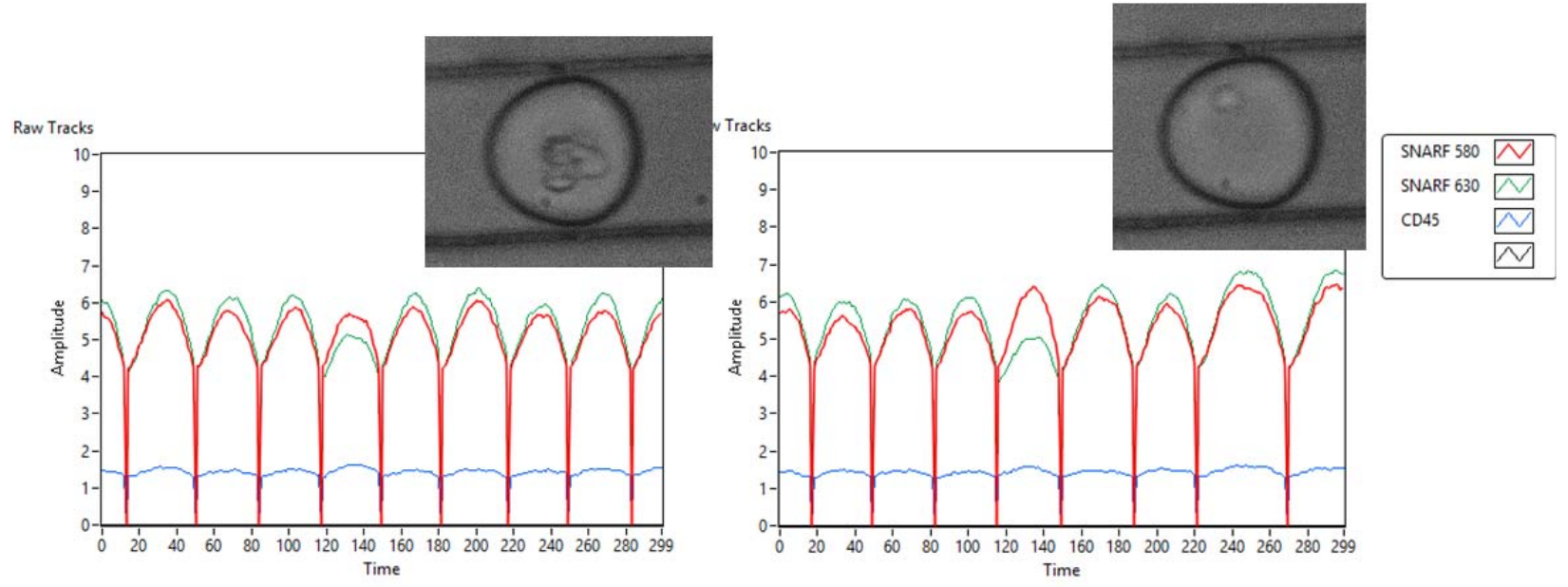

Figure S11-B. CD45(-) cluster, and small CD45(-) cell. 


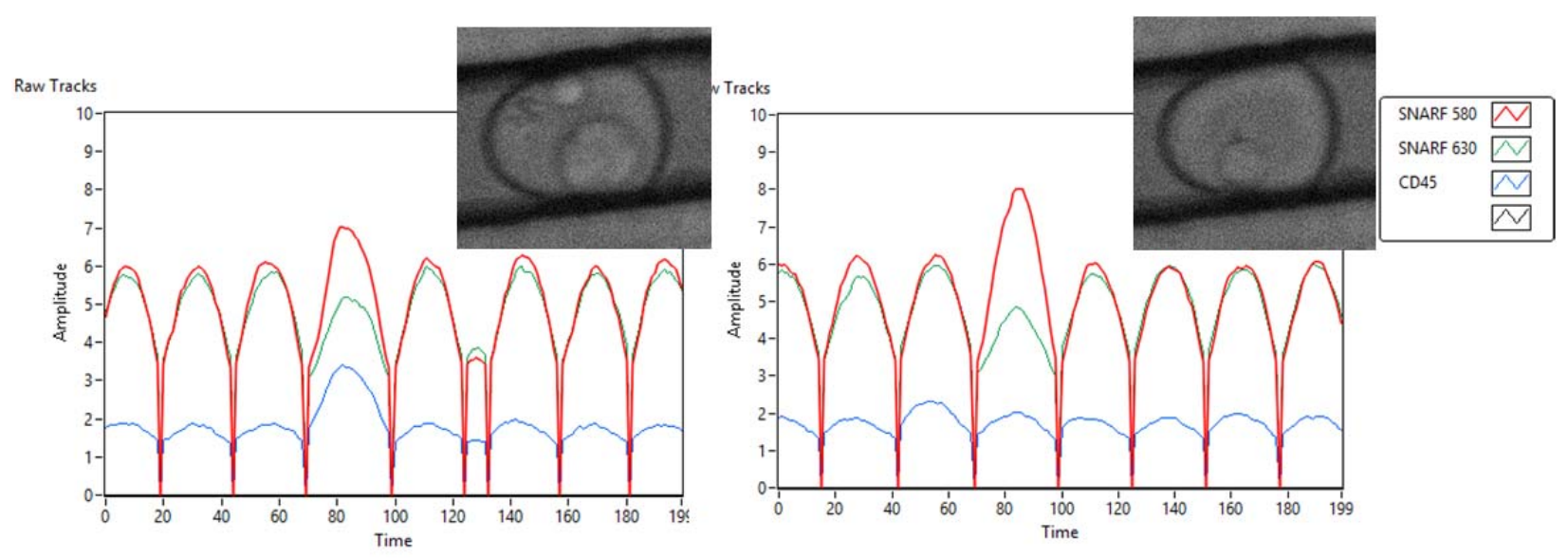

Figure S11-C. Big cells (20.8um) incompatible with white blood cell size (note droplet positivity to CD45 due to another WBCS enclosed together) and another CD45(-) cell.

\section{Patient statistics}

The table reports patient statistics compared to healthy donors. Sample were depleted from CD45(+) cells with magnetic depletion and stained with CD45 antibody. Data reported are normalized for 1000000 drops. Total droplet acquired for each sample ranges from 700,000 to 1,500,000.

\begin{tabular}{|l|l|l|}
\hline CD45(-)Acid $(+)$ cells $/ 10^{6}$ drops & Cancer patient & Healthy donor \\
\hline Lung & 9 & 0 \\
\hline Lung & 20 & 1 \\
\hline Breast & 32 & 0 \\
\hline Pancreas & 5 & 0 \\
\hline
\end{tabular}

Table S1 - Patient statistics compared to healthy donors.

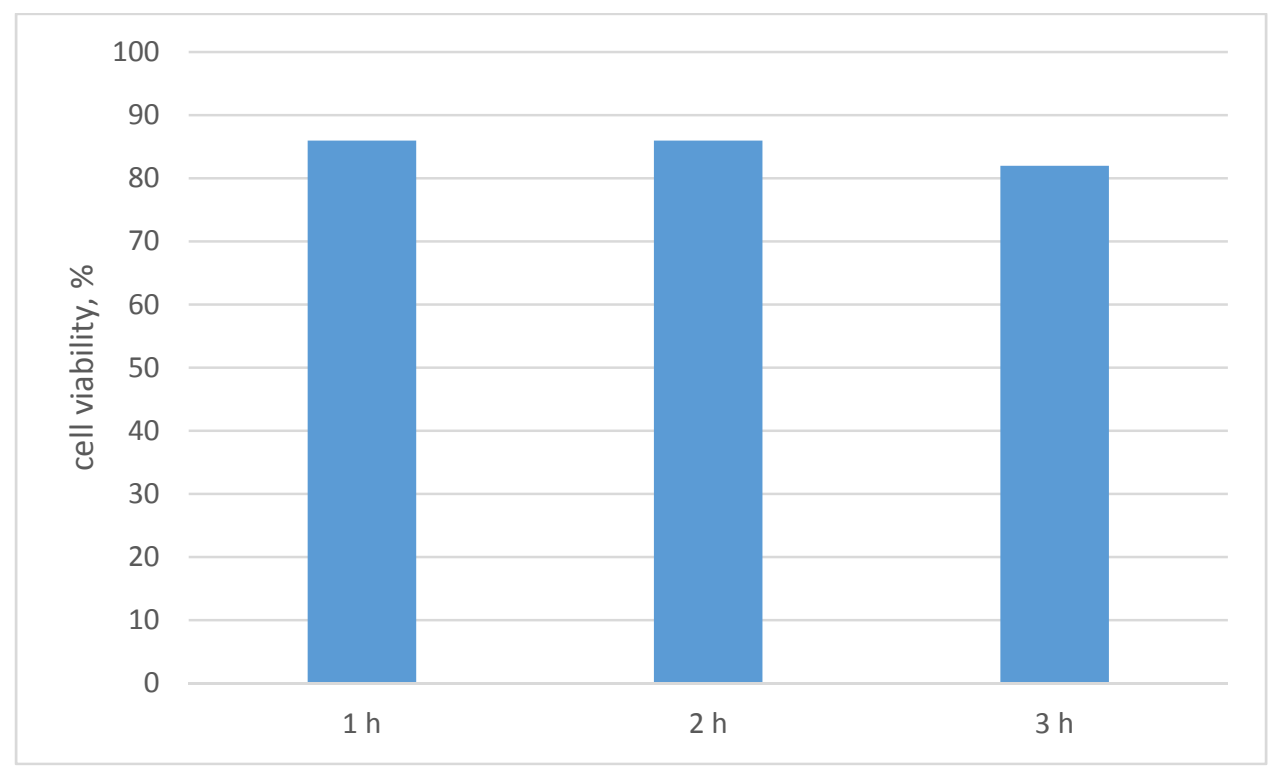


Figure S12. Viability assay. A549s were emulsified in running buffer (2\% BSA in Joklik modification medium, $\mathrm{pH}$ 7.4) and incubated at $37 \mathrm{C}$ for specified period of time. After breaking emulsion cells were stained with Calcein AM and SYTOX AADvanced. Cells positive for Calcein AM considered as live, SYTOX ADDvanced positive cells considered as dead irrespective of Calcein AM response. 